ORIGINAL PAPER

\title{
Prevalence of the Quilty effect in endomyocardial BIOPSY OF PATIENTS AFTER HEART TRANSPLANTATION - FROM CELLULAR REJECTION TO ANTIBODY-MEDIATED REJECTION?
}

\author{
Sylwia Szymanska ${ }^{1}$, Wieslawa Grajkowska ${ }^{1}$, Malgorzata Sobieszczanska-Malek ${ }^{2}$, \\ Tomasz Zielinski ${ }^{2}$, Michal Pyzlak ${ }^{1,3}$, Maciej Pronicki ${ }^{1}$ \\ ${ }^{1}$ Department of Pathology, The Children's Memorial Health Institute, Warsaw, Poland
2Department of Heart Failure and Transplantation, The Institute of Cardiology, Warsaw, Poland
${ }^{3}$ Department of General and Experimental Pathology, Medical University of Warsaw, Warsaw, Poland
}

The significance of the Quilty effect $(\mathrm{QE})$ is not fully understood. It was once proposed to be related to acute cellular rejection (ACR). We aim to assess the relation between QE prevalence and antibody-mediated rejection (AMR).

One thousand three hundred and fifty endomyocardial biopsies (EMBs) from 212 patients who underwent heart transplantation in the years 2001-2013 and survived a period of 30 days after the operation were diagnosed. In all EMBs routine HE staining and additional immunohistochemical staining with polyclonal antibody against $\mathrm{C} 4 \mathrm{~d}$ were performed. Microscopic findings were classified according to the new ISHLT 2013 criteria. Patients were separated into two groups: group 1 included those with at least one pAMR1 I+ and/or pAMR2 EMB ( $n=16)$, and group 2 included the rest of the patients $(\mathrm{n}=196)$. Presence of $\mathrm{QE}$ with distinguishing subtypes A and B (according to the first ISHLT 1990 criteria) was assessed.

One hundred and twenty one EMBs from group 1 and 1229 EMBs from group 2 were diagnosed. Quilty effect type A was found in 16(13.2\%) EMBs in group 1 and in $96(7.8 \%)$ EMBs in group 2, $\mathrm{p}<0.001$. Quilty effect type B was diagnosed in 52 (43\%) EMBs in group 1 and in $245(20 \%)$ EMBs in group 2, $p<0.001$. The QE was not present in $53(43.8 \%)$ EMBs in group 1 and in 888 (72.2\%) EMBs in group 2, $\mathrm{p}<0.001$.

The relation between $\mathrm{QE}$ prevalence and $\mathrm{AMR}$ is possible as the $\mathrm{QE}$ is present statistically more often in EMBs of patients with C4d capillary depositions.

Key words: heart transplantation, antibody-mediated rejection, Quilty effect, C4d.

\section{Introduction}

The Quilty effect (QE) was first described by Billingham in 1981. It was defined as a lymphocytic infiltrate bulging in the endocardium of a cardiac allograft with or without involvement of the myocardium [1]. The clinical significance and mechanism of this lesion are still controversial. Quilty effect inci- dence in endomyocardial biopsies (EMBs) is also not established - according to some authors it varies from $10 \%$ to $20 \%$ [2]. The first International Society for Heart and Lung Transplantation (ISHLT) criteria for reporting heart allograft rejection (ISHLT 1990) [3] classified the QE into two subtypes based on its relation to myocardium. Subtype A was supposed to be 
limited to the endocardium while subtype B involved the myocardium. Later on, the clinical significance of distinct types was questioned and the new ISHLT 2004 criteria did not sustain the QE classification [4]. In the early 1990s the QE was believed to be related to cyclosporine therapy [5]. A drug toxic effect or immunologic reaction to this immunomodulator was proposed as a potential trigger. Further studies [6, 7] did not prove that hypothesis as the lesion had been found in EMBs of patients treated with different kinds of immunomodulators. Other authors [8, 9] believed that the $\mathrm{QE}$ was a kind of low-grade cellular rejection (ACR), specially type B. The potential mechanism was separation of inflammatory infiltrates from the endocardium. Nowadays, this relation seems unlikely, yet some studies in recent years have shown a greater possibility of developing rejection in patients who manifest the QE [2]. Recent studies showed that IL-10 gene polymorphism, associated with decreased cytokine production, was increased in patients with Quilty lesions [10]. It was also proved that the QE had features of a tertiary lymphoid tissue [11]. Most recently, QE areas were reported to be frequently associated with endocardial C4d deposition [12]. C4d is a well-known immunohistochemical marker of antibody-mediated rejection (AMR). Humoral rejection remains a significant factor of unfavorable allograft function and survival. Therefore, the aim of this study was to assess the relation between QE prevalence and AMR.

\section{Material and methods}

One thousand three hundred and fifty EMBs from 212 patients who underwent heart transplantation in the years 2001-2013 and survived a period of 30 days after the operation were enrolled in the study. All EMBs were retrospectively examined in the Department of Pathology, The Children's Memorial Health Institute, by three qualified pathologists unaware of the clinical data. Endomyocardial specimens were taken from the right ventricle, fixed in $4 \%$ formalin and embedded in paraffin. The paraffin blocks were cut into $4 \mu \mathrm{m}$ slices and routinely stained with hematoxylin/eosin (HE). Immunohistochemical (IHC) staining with polyclonal antibodies against $\mathrm{C} 4 \mathrm{~d}$ (Biomedica Gruppe, dilution 1 : 40) was performed in all diagnostic EMBs as the marker of antibody-mediated rejection (AMR). Features indicative of AMR were summarized as capillary deposition of C4d of the myocardium (> $50 \%$ of vessels involved). The staining of venular, arterial, or arteriolar endothelial cells, arterial elastic lamina, and the capillaries in the $\mathrm{QE}$ were not considered to be indicative of AMR. CD68 stain was not performed in our department. Microscopic findings were classified according to the new ISHLT 2013 classification. Patients were divided into two groups based on the $\mathrm{C} 4 \mathrm{~d}$ reaction. Group 1 included those with at least one pAMR1 I+ and/ or pAMR2 EMB ( $\mathrm{n}=16)$, and group 2 included the rest of the patients $(n=196)$. Presence of $Q E$ with distinguishing subtypes $\mathrm{A}$ and $\mathrm{B}$ (according to the first ISHLT 1990 criteria) was assessed. Information concerning donor-specific antibody (DSA) presence was not available for all patients. For statistical analysis of the result Statistica software was used and the $\chi^{2}$ test was applied.

\section{Results}

One hundred and twenty one EMBs from 16 patients from group 1 and 1229 EMBs from 196 patients from group 2 were diagnosed. In group 1 pAMR1I+ was present in 12 EMBs $(9.9 \%)$ and pAMR2 in 11 EMBs (9.1\%). Quilty effect type A was found in $16(13.2 \%)$ EMBs in group 1 and in $96(7.8 \%)$ EMBs in group 2, $\mathrm{p}<0.001$. Quilty effect type B was diagnosed in $52(43 \%)$ EMBs in group 1 and in $245(20 \%)$ EMBs in group 2, p $<0.001$. The QE was not present in $53(43.8 \%)$ EMBs in group 1 and in $888(72.2 \%)$ EMBs in group 2, p $<0.001$. There was no difference in the frequency of each $\mathrm{QE}$ subtype between groups in AMR-positive biopsies. Results are summed up in Table I.

\section{Discussion}

The significance and clinical implications of the QE are still not entirely clear although 34 years have passed since the first description of the lesion. The greatest literature impact on the QE comes from the 1990s, when its inflammatory composition and potential mechanism were studied. Some authors connected the QE with cyclosporine therapy [5], the QE being thought to be a kind of ACR $[8,9]$. Most of these findings are no longer sustained. In EMBs of patients treated with modern immunomodulators the $\mathrm{QE}$ is found as frequently as on previous therapy.

Table I. Prevalence of Quilty effect in patients with and without C4d positivity

\begin{tabular}{lcc}
\hline Quilty effect $(\mathrm{QE})$ & C4D $(+)$ EMBs $(\mathrm{N}=121)$ & C4D $(-)$ EMBs $(\mathrm{N}=1229)$ \\
\hline Any subtype & $68(56.2 \%)$ EMBs & $341(27.8 \%)$ EMBs \\
\hline QE type A & $16(13.2 \%)$ EMBs & $96(7.8 \%)$ EMBs \\
\hline QE type B & $52(43 \%)$ EMBs & $245(20 \%)$ EMBs \\
\hline
\end{tabular}



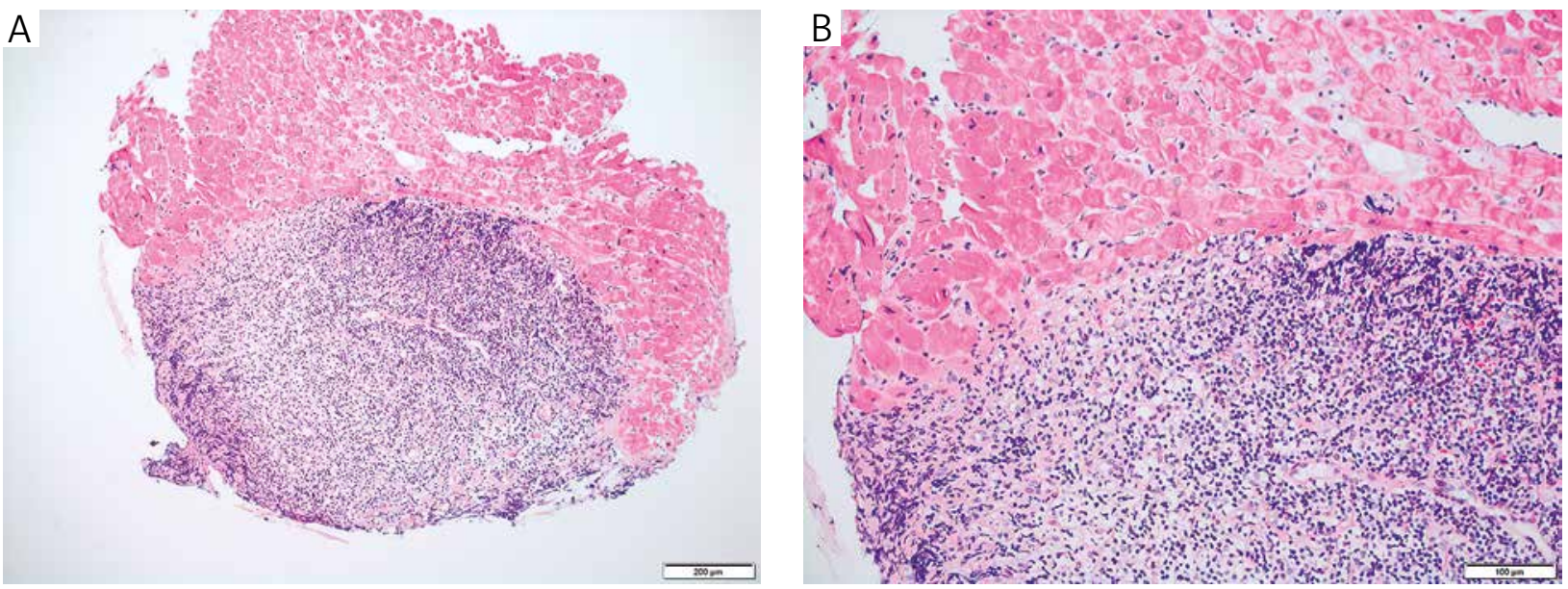

Fig. 1. Quilty effect type A. In the image extensive lymphocytic infiltrate bulging in the endocardium is present. Although the lesion is wide the myocardium is not involved. A) HE staining, original magnification $200 \times$; B) HE staining, original magnification $400 \times$

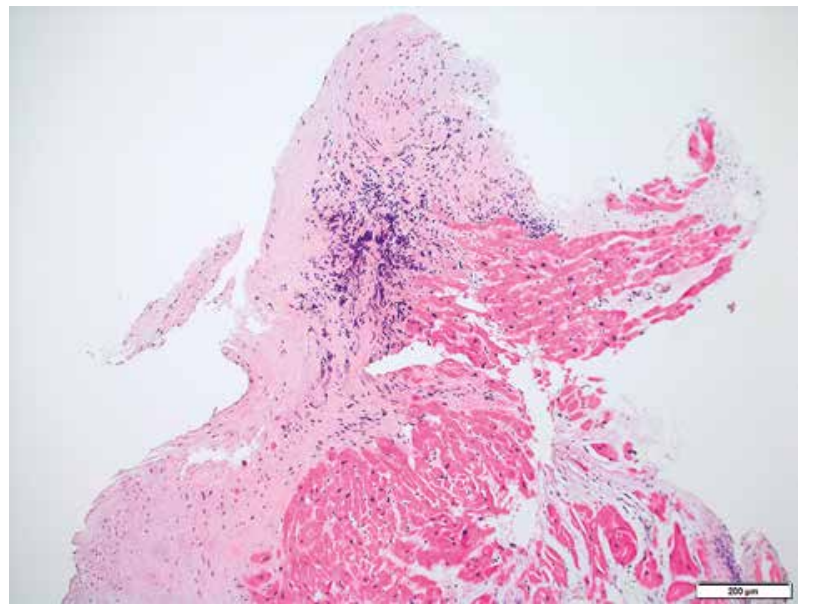

Fig. 2. Quilty effect type B. Lymphocytic infiltrate is moderate but it involves the myocardium. Prominent endocardial fibrosis is also present. HE staining, original magnification $200 \times$
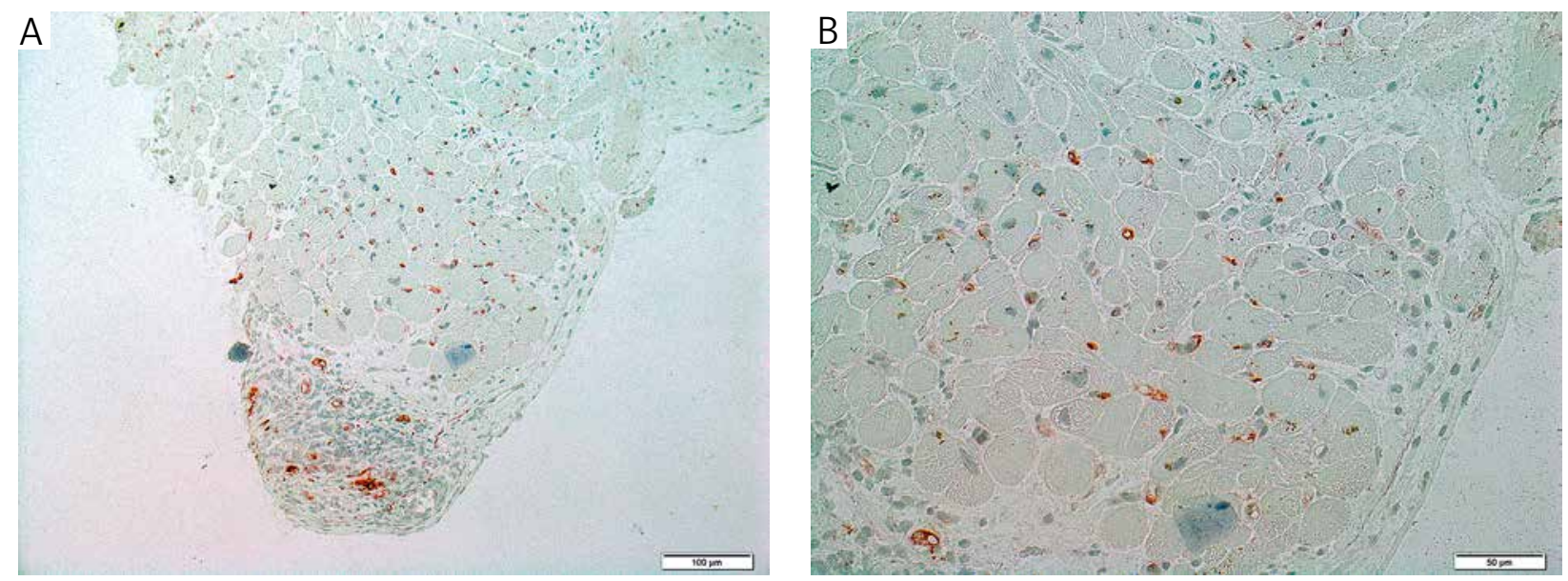

Fig. 3. C4d positive staining in EMB of patient with Quilty effect: A) original magnification $200 \times$; B) original magnification $400 \times$ 
whether there is any difference in terms of humoral rejection. No difference between the subtypes was found. The obtained results proved that QE classification is justifiable neither from a clinical nor a histopathological point of view. For analysis we chose the chi-squared test because it is the most appropriate tool to present independent normally distributed data. Our study was a retrospective one and initial microscopic diagnosis was present in previous ISHLT criteria; therefore we reclassified all EMBs according to the new ISHLT 2013 criteria. Significance of histopathological changes in terms of humoral rejection is still discussed. Our preliminary data [14] proved that microscopic features suggestive of AMR are found relatively often in EMBs of patients after heart transplantation, regardless of $\mathrm{C} 4 \mathrm{~d}$ positive stain, and we would like to discuss that matter in a separate paper. In this study we focused on complement depositions in capillaries, as they are believed to be strongly connected with AMR. Therefore we isolated patients who have had at last one EMB with diagnosis of pAMR1 I+ and/or pAMR2. The examination proved that $\mathrm{QE}$ was observed statistically more frequently in that group than in the rest of the patients. Concomitant presence of $\mathrm{QE}$ and $\mathrm{C} 4 \mathrm{~d}$ deposition was found in 10/121 EMBs (8.3\%), but lesions were often observed in both previous and subsequent biopsies of those patients. Thus, we concluded that a common mechanism of $\mathrm{QE}$ and complement deposition development may exist. Nonetheless, the correlation remains rather weak, which may suggest a multifactorial rather than a simple direct relationship. Cano et al. [12] published similar observations, but their background was different than ours. They analyzed the $\mathrm{C} 4 \mathrm{~d}$-positive linear staining in the endocardium surrounded by the lymphocytes forming the QE lesion. In some cases, the linear $\mathrm{C} 4 \mathrm{~d}$ deposition extended to the endocardium surrounding the $\mathrm{QE}$ area. In their material there were no signs of rejection. In our department, we also sometimes observe linear C4d positivity in the endocardium, but we interpret it as non-specific staining. Cano et al. suggested that the concomitant presence of $\mathrm{QE}$ and $\mathrm{C} 4 \mathrm{~d}$ depositions in an allograft heart biopsy, without evidence of rejection, indicated better adaptation of the allograft to the host ("accommodation") [12]. Our experience supports that hypothesis - most of the C $4 \mathrm{~d}$-positive patients have never presented any clinical symptoms. Preliminary clinical analysis proved that there were no statistically significant differences in survival between patients with positive staining and without $\mathrm{C} 4 \mathrm{~d}+$ staining when Kaplan-Meier survival curves were compared. Obviously, there is still a lot of controversy on the issue. We do not fully understand the mechanism of AMR itself, so it is difficult to propose a common pathogenic pathway between humoral rejection and QE. Most likely, different factors, which should be further explored, induce coexistence of Quilty lesions and AMR. A major limitation of our study is the lack of DSA detection in all suspected AMR patients. It may be explained by the retrospective character of the study. Most EMBs were archival, diagnosed before 2004. Unfortunately, serum was not preserved in any case and we could not perform the tests. Besides, DSA testing is relatively expensive, and it is not possible to verify it at all times. Nonetheless, the relation between Quilty effect prevalence and AMR may be possible. To the best of our knowledge, our study is the second one to imply a relation between C4d deposition and the QE. Further studies on larger groups of patients are mandatory.

\section{Conclusions}

The relation between QE prevalence and AMR is possible as the QE is present statistically more often in EMBs of patients with C4d-positive staining.

\section{The authors declare no conflict of interest.}

\section{References}

1. Mohacsi PJ, Joshi A, Wang J, et al. Endocardial mononuclear cell infiltrates (Quilty effect) in heterotopic cardiac allografts in rapamycin-treated rats. Transplant Proc 1994; 26: 3255-3259.

2. Mozaffari K, Bakhshandeh H, Amin A, et al. Diagnostic pitfalls and challenges in interpretation of heart transplantation rejection in endomyocardial biopsies with focus on our experience. Res Cardiovasc Med 2014; 3: e13986.

3. Billingham ME, Cary NRB, Hammond EH, et al. A working formulation for the standardization of nomenclature in the diagnosis of heart and lung rejection: Heart Rejection Study Group. J Heart Transplant 1990; 9: 587-593.

4. Stewart S, Winters GL, Fishbein MC, et al. Revision of the 1990 working formulation for the standardization of nomenclature in the diagnosis of heart rejection. J Heart Lung Transplant 2005; 24: 1710-1720.

5. Forbes RD, Rowan RA, Billingham ME. Endocardial infiltrates in human heart transplants: a serial biopsy analysis comparing four immunosuppression protocols. Hum Pathol 1990; 21: 850-855.

6. Freimark D, Czer LS, Aleksic I, et al. Pathogenesis of Quilty lesion in cardiac allografts: relationship to reduced endocardial cyclosporine A. J Heart Lung Transplant 1995; 14: 11971203.

7. Barone JH, Fishbein MC, Czer LS, et al. Absence of endocardial lymphoid infiltrates (Quilty lesions) in nonheart transplant recipients treated with cyclosporine. J Heart Lung Transplant 1997; 16: 600-603.

8. Chantranuwat C, Blakey JD, Kobashigawa JA, et al. Sudden, unexpected death in cardiac transplant recipients: an autopsy study. J Heart Lung Transplant 2004; 23: 683-689.

9. Pardo-Mindán FJ, Lozano MD, Contreras-Mejuto F, de Alava E. Pathology of heart transplant through endomyocardial biopsy. Semin Diagn Pathol 1992; 9: 238-248.

10. Plaza DM, Fernández D, Builes M, et al. Cytokine gene polymorphisms in heart transplantation: association of low IL-10 production genotype with Quilty effect. J Heart Lung Transplant 2003; 22: 851-856. 
11. Di Carlo E, D'Antuono T, Contento S, et al. Quilty effect has the features of lymphoid neogenesis and shares CXCL13-CXCR5 pathway with recurrent acute cardiac rejections. Am J Transplant 2007; 7: 201-210.

12. Cano LC, Arteta AA, Fernandez R, et al. Quilty effect areas are frequently associated with endocardial C4d deposition. J Heart Lung Transplant 2008; 27: 775-779.

13. Zakliczynski M, Nozynski J, Konecka-Mrowka D, et al. Quilty effect correlates with biopsy-proven acute cellular rejection but does not predict transplanted heart coronary artery vasculopathy. J Heart Lung Transplant 2009; 28: 255-259.

14. Szymanska S, Grajkowska W, Sobieszczanska-Malek M, et al. Reclassification of C4d-Positive Endomyocardial Biopsy (EMB) According to New International Society for Heart and Lung Transplantation (ISHLT) 2013 Categories for Reporting Pathologic Antibody-Mediated Rejection (pAMR): Preliminary Data from a Polish Single-Center Study. Ann Transplant 2015; 20: 351-356.

\section{Address for correspondence}

Sylwia Szymanska

Department of Pathology

The Children's Memorial Health Institute

Al. Dzieci Polskich 20

04-730 Warsaw, Poland

tel. +48228151960

fax +48228151975

e-mail: s.szymanska@ipczd.pl 